\title{
Fluxes and energy dissipation in thermal convection and shear flows
}

\author{
Bruno Eckhardt ${ }^{1}$, Siegfried Grossmann ${ }^{1}$ and Detlef Lohse $^{2}$ \\ 1 Fachbereich Physik, Philipps-Universität Marburg, D-35032 Marburg, Germany \\ 2 Department of Applied Physics, University of Twente, 7500 AE Enschede, \\ The Netherlands
}

PACS. 47.55.P- - Buoyancy-driven flows; convection.

PACS. 47.55.pb - Thermal convection.

PACS. 47.20.Qr - Centrifugal instabilities.

PACS. 47.27.nd - Channel flow.

\begin{abstract}
We expose analogies between turbulence in a fluid heated from below (RayleighBénard (RB) flow) and shear flows: The unifying theory for RB flow (S. Grossmann and D. Lohse, J. Fluid Mech. 407, 27-56 (2000) and subsequent refinements) can be extended to the flow between rotating cylinders (Taylor-Couette flow) and pipe flow. We identify wind dissipation rates and momentum fluxes that are analogous to the dissipation rate and heat flux in RB flow. The proposed unifying description for the three cases is consistent with the experimental data.
\end{abstract}

Net transport of heat, momentum, and kinetic energy in turbulent flows arises in many practical situations. The determination of its magnitude poses a formidable challenge to our understanding of the dynamics of turbulence. In the absence of an exact theory, various approximations, prominent among them the Prandtl mixing layer model, have been proposed for its modelling. Where no absolute numbers could be obtained, theory has aimed to predict at least the scaling with Reynolds number or other system parameters. In the case of heat transport in a layer of fluid heated from below considerable insights have been gained from an ansatz that combines two exact equations for the heat current and the energy dissipation rate with scaling assumptions for the behaviour in plume dominated boundary layers and fluctuation dominated bulk regions [1]. In this way the complete range of responses of a fluid to a temperature gradient can be determined. Specifically, with the Prandtl number characterizing the fluid properties and the Rayleigh number as the external control parameter, the response in the form of a fluid flow, measured by a wind (or transverse) velocity $R e_{w}$, and a thermal transport, described by the Nusselt number $N u_{\theta}$, can be predicted and different regions in the $N u_{\theta}(R a, P r)$ and $R e_{w}(R a, P r)$ plane can be identified. A considerable body of experimental data is in very good agreement with the predictions and has confirmed the basic scaling assumptions of the theory. The quality of the data has improved to the point that influences from the side walls [2] and the top and bottom plates [3-5], due to finite conductivity of the solid boundary materials, or deviations from the Boussinesq approximation [6] can be identified.

(c) EDP Sciences 

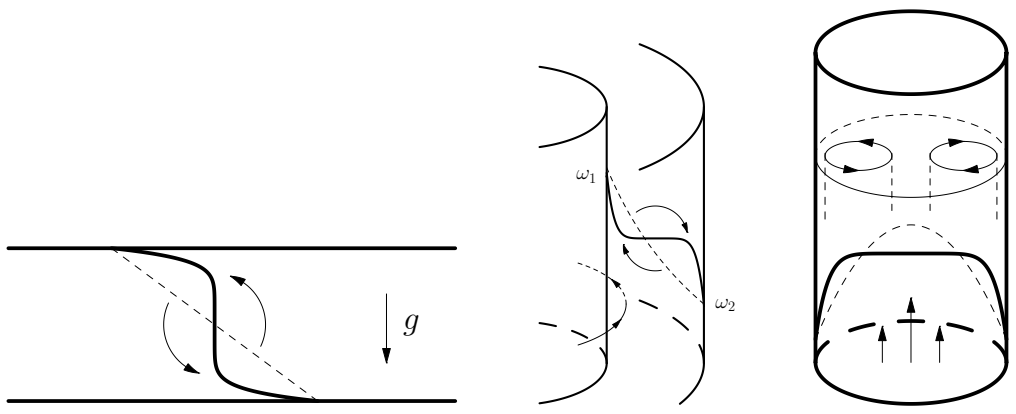

Fig. 1 - Comparison between (a) Rayleigh-Bénard, (b) Taylor-Couette, and (c) pipe flow, arranged so as to highlight the similarities of the flows. The lengths scale is set by the gap width $d$ in RB and TC flow and the radius $a$ in pipe flow. In the RB case we show the lower warm and upper cold plates, and the mean temperature profile. The deviation from the linear profile is due to heat transport by a transverse velocity field (the wind) that has components in the direction of the thermal gradient and, by Navier-Stokes interaction, also in the perpendicular directions. In the TC case the corresponding profile is one of angular velocity. Deviations from the (slighly curved) laminar profile are due to angular velocity transport by a velocity in radial direction ("Taylor vortices") and, again by Navier-Stokes interaction, in axial and azimuthal directions. For pipe flow, we show the mean axial velocity, which is again deformed by wind contributions in the radial and consequently in azimuthal and axial directions. A fourth flow of similar geometry and transverse transport would be pressure driven Poiseuille flow between flat plates.

In the case of shear flows, in particular in confined geometries like pipe and Taylor-Couette (TC) flows, the theory is less well developed. Extensions of the Rayleigh-Bénard reasoning to Taylor-Couette and pipe flow have been proposed in [7] and in [8]. However, in both references the analogy was incomplete and additional fit parameters or approximations had to be introduced. This state of affairs is particularly irritating for TC flow, where there is a one-to-one relation with RB flow in its linear stability properties, and where various analogies for the fully nonlinear case have been discussed in the literature [8-11]. However, none of these analogies is exact in the full 3-dim nonlinear case. Our aim here is to remedy this situation and to present pairs of quantities in the fully nonlinear Rayleigh-Bénard, Taylor-Couette, and pipe flow geometries that are dynamically related in a one-to-one fashion. This translation opens up new avenues for the analysis of shear flows, suggests scaling behaviour in close analogy to what has been confirmed in RB flow, and raises several questions about the dynamics of shear flows.

In order to outline the similarities between the three flows, we begin by recalling the essential steps of the analysis of RB flow. For the time being we neglect various details, like side wall influences etc, and focus on the dominant behaviour. As the analysis of the existing data for TC flow given below will show, their precision is as yet insufficient to clearly identify and study such corrections.

Rayleigh-Bénard flow: In the case of a fluid layer heated from below the 'laminar' state is one without fluid flow, $\mathbf{u}=0$, and a linear temperature drop from bottom to top plate. In this state there is only molecular transport of heat. The flow that sets in once a critical temperature difference is exceeded, the 'wind', increases the heat flux by advection of heat (Fig. 1). The total heat flux can be obtained from the equation for the temperature field by averaging over planes $A$ parallel to the bottom and top plates (and time $t$ ). With $z$ the coordinate normal to the plates, we find the expression for the conserved, $z$-independent heat 
flux

$$
J_{\theta}=\left\langle u_{z} \theta\right\rangle_{A, t}-\kappa \frac{\partial\langle\theta\rangle_{A, t}}{\partial z}=N u_{\theta} J_{\theta, l a m} .
$$

The last equality defines the Nusselt number $N u_{\theta}$ as the ratio of the actual heat current to the laminar value $J_{\theta, \text { lam }}=\kappa \Delta / d$, where $\kappa$ is the thermal conductivity, $\Delta$ the imposed temperature difference, and $d$ the height of the box. The kinetic energy in the wind is dissipated by fluid viscosity $\nu$. Multiplying the Navier-Stokes equations with the velocity $\mathbf{u}$ and integrating over the volume, the energy balance between dissipation and input reads

$$
\epsilon_{w}=\nu^{3} d^{-4} \operatorname{Pr}^{-2} R a\left(N u_{\theta}-1\right) .
$$

Here, $\operatorname{Pr}=\nu / \kappa$ is the Prandtl number and $R a=\alpha_{p} g d^{3} \Delta /(\kappa \nu)$ the Rayleigh number. These two relations, (1) and (2), show the intimate relation between the forcing by an externally applied profile in one of the fields, here the temperature field, and the wind: there is only diffusive transport if there is no wind, and there can be no wind unless there is an increased energy uptake $N u_{\theta}>1$.

In case of $\mathrm{RB}$ flow two dissipation rates can be calculated: a dissipation rate $\epsilon_{w}$ for the velocity field from the $\mathbf{u}$-equation, and a thermal dissipation $\operatorname{rate} \epsilon_{\theta}=\kappa\left\langle\operatorname{grad}^{2} \theta\right\rangle$ from the temperature equation. However, $\epsilon_{\theta}$ carries the same information as the thermal current $J_{\theta}=N u \kappa \Delta / d$, since $\epsilon_{\theta}=\kappa \Delta^{2} d^{-2} N u$. In TC and pipe flow one again can derive a wind dissipation rate $\epsilon_{w}$ from the Navier-Stokes equation for the velocity field vector $\mathbf{u}$, but one does not succeed in identifying another dissipation rate for the one velocity component which has changed its profile because of the externally applied boundary conditions. However, the presence of a profile already in the laminar state shows that there is a transverse transport of this quantity and the associated conserved current can be generalized also to the turbulent situation. Thus, we will built our generalization to other flows on the pair formed by wind dissipation $\epsilon_{w}$ and current $J$.

Taylor-Couette flow: The flow between two independently rotating cylinders (Fig. 1b) has several parameters, including the gap width $d=r_{2}-r_{1}$, the radius ratio $\eta=r_{1} / r_{2}$, and the angular velocities $\omega_{1}$ and $\omega_{2}$ of the inner and outer cylinders. In the laminar case the velocity field is azimuthal, $\mathbf{u}=r \omega(r) \mathbf{e}_{\phi}$; its shape is close to a linear interpolation between the angular velocities in the small gap limit $\eta \rightarrow 1$. This suggests to consider the angular velocity and the radial direction as corresponding to the temperature profile and the normal direction in the RB situation. Taking the $\phi$-component of the Navier-Stokes equation and averaging over cylinders at fixed $r$ between $r_{1}, r_{2}$, we find that the quantity

$$
J_{\omega}=r^{3}\left(\left\langle u_{r} \omega\right\rangle_{A, t}-\nu \partial_{r}\langle\omega\rangle_{A, t}\right)
$$

is independent of the radius and hence conserved, also in the turbulent case. It corresponds to the heat current in the RB case. The appearance of the angular velocity rather than angular momentum or azimuthal velocity is unexpected, but dictated by the viscous part: The NavierStokes equation implies that it is only for this combination that the viscous part is the radial derivative of a mean profile. In laminar TC flow only the second term contributes,

$$
J_{\omega, l a m}=\nu r_{1}^{2} r_{2}^{2} r_{a}^{-1} d^{-1}\left(\omega_{1}-\omega_{2}\right) .
$$

$r_{a}=\left(r_{1}+r_{2}\right) / 2$ denotes the average radius and $d=R_{2}-r_{1}$ the gap width. Deviations from the laminar profile require the presence of a radial velocity contribution $u_{r}$, which plays the role of the wind in the RB case. Since the reference laminar flow already has a shear profile 
that dissipates energy, we consider the difference in dissipation between the case with wind and the case without,

$$
\epsilon_{w}=\epsilon-\epsilon_{\text {lam }}=r_{a}^{-1} d^{-1}\left(\omega_{1}-\omega_{2}\right) J_{\omega, l a m}\left(N u_{\omega}-1\right) .
$$

The fluid is driven by rotating the cylinder walls with angular velocities $\omega_{1}$ and $\omega_{2}$, respectively, and it responds with an angular velocity transport $N u_{\omega}=J_{\omega} / J_{\omega, \text { lam }}$ and by setting up a wind ("Taylor vortices") with amplitude $U_{w}$ that can be characterized by a Reynolds number $R e_{w}=U_{w} d / \nu$. In analogy to RB flow, the aim then is to predict $N u_{\omega}$ and $R e_{w}$ and their dependencies on the angular velocities $\omega_{1}$, and $\omega_{2}$, the radius ratio $\eta$, the aspect ratio, or other parameters.

Pipe flow: Laminar pressure driven flow with mean velocity $U$ and Reynolds number $R e=U 2 a / \nu$ down a pipe of radius $a$ shows a parabolic profile of the axial velocity $u_{z}$ (Fig. 1c). In the turbulent case this profile is modified by a radial flow $u_{r}$, which the NavierStokes equation couples to an azimuthal and an additional axial velocity field. Averaging the Navier-Stokes equation for the axial velocity $u_{z}$ over axial cylinders $r=$ const we can identify

$$
J_{u_{z}}=\frac{1}{r}\left(\left\langle u_{r} u_{z}\right\rangle_{A^{(=), t}}-\nu \partial_{r}\left\langle u_{z}\right\rangle_{A^{(=), t}}\right)
$$

as the conserved transverse $u_{z}$-flux. The laminar flux is

$$
J_{u_{z}, l a m}=8 \nu a^{-2} U=4 \nu^{2} a^{-3} R e .
$$

Beyond laminarity there is a wind of amplitude $U_{w}$ whose radial component $u_{r}$ enhances the $u_{z}$-transport by convection. Near the threshold for the transition to turbulence this wind is dominated by downstream vortices similar to the Taylor-vortices in TC flow $[12,13]$. Its dissipation rate can be evaluated as

$$
\epsilon_{w}=\epsilon_{u}-\epsilon_{l a m}=U J_{u_{z}, l a m}\left(N u_{u_{z}}-1\right),
$$

with the nondimensionalized transverse flux "Nusselt" number $N u_{u_{z}}=J_{u_{z}} / J_{u_{z}, l a m}$.

The analogy between the three examples is evident: in all cases we have a conserved current $J$ and a dissipation rate for the wind $\epsilon_{w}$. The link between each $J$ and the respective $\epsilon_{w}$ is an intimate one, in that one cannot have an increase in the current without a non-zero dissipation rate of the wind and hence, in the statistically stationary case, in the power input. The expressions for the fluxes $J$ and the wind dissipation rates $\epsilon_{w}$ as well as the relations (2), (5), and (8) between them are exact consequences of the Navier-Stokes equations.

Scaling relations: Proceeding as in the case of RB thermal convection [1], we model - e.g. for TC flow - the energy dissipation rate of the wind as the superposition of the dissipation in the boundary layer plus that in the bulk,

$$
2 \operatorname{Re}^{2}(N u-1)=\frac{\epsilon_{w}}{\nu^{3} d^{-4}}=c_{1} R e_{w}^{5 / 2}+c_{2} R e_{w}^{3} .
$$

The flux consists of a boundary layer and plume (hairpin) contribution plus a bulk or fluctuation contribution

$$
N u=c_{3} \sqrt{R e_{w} g}+c_{4} R e_{w} g .
$$

The switching function $g(s)$ depends on the ratio $s=\sqrt{R e_{w}} / N u \propto \lambda / \delta$ of the thickness $\lambda$ of the profile forming quantity (temperature, angular velocity or axial velocity) and that of the wind boundary layer $\delta$. It accounts for the fact that the velocity that determines the 

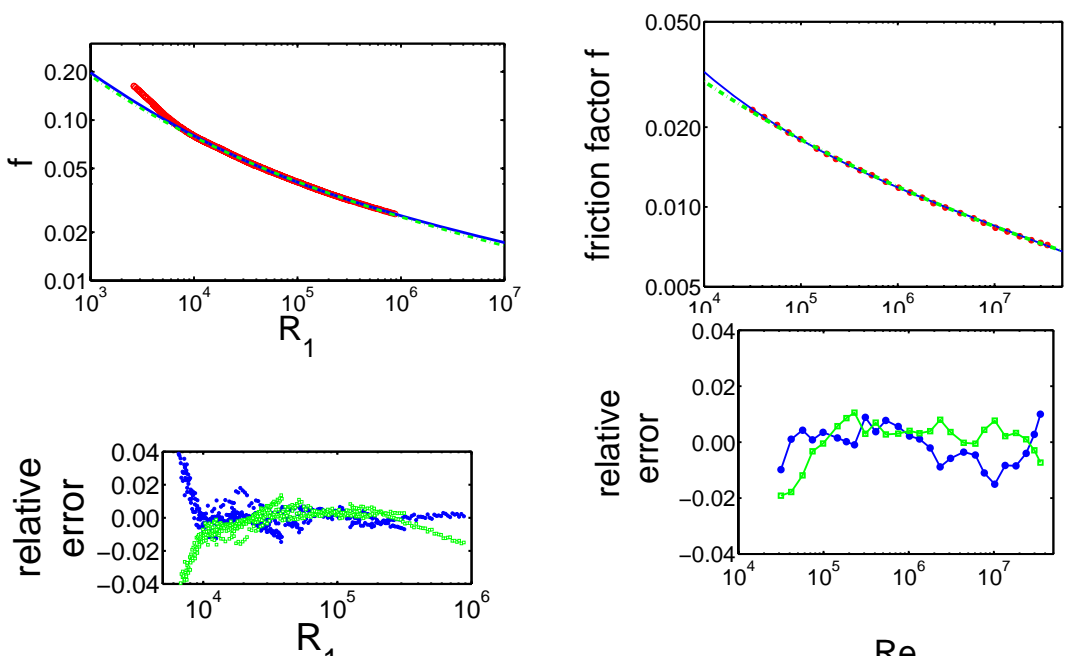

Fig. 2 - Friction factor $1 \mathrm{~s}$. Reynolds number for TC flow (left) $\mathrm{Re}$ and pipe flow (right). For TC-flow the data are from [17] and for pipe flow from [19]. The top frame shows the friction factors for the data (red symbols), the fit based on eqs. $(9,10,11)$ (full blue line) and the Prandtl-von Karman law (dash-dotted green line). The lower frames show the relative errors.

momentum transport has to be adjusted (a detailed discussion in the context of RB flows is given in Ref. 1c). If the boundary layer for the wind is thinner than the one for the driving field, $\delta<\lambda$, then the full velocity has to be taken, $g(s)=1$. If the boundary layer for the wind is wider, than only the gradient that can be built up over the width $\lambda$ matters, $g(s)=s$. Most experiments on RB flows are typically in either one or the other regime or switch quickly between them [1]. However, guided by the observation in [7] that even for the largest Reynolds numbers accessible no pure scaling regime can be found, we adopt for the switching function - slightly different from the one used in ref. 1c - the form

$$
g(s)=s^{2+\gamma} /\left(c_{5}+s\right)
$$

This definition completes our modelling; equations (9), (10), and (11) together now form a nonlinear, implicit system for the wind Reynolds number $R e_{w}$ and the Nusselt number $N u$.

Without a point of reference for the wind Reynolds number, the four coefficients $c_{1}, c_{2}$, $c_{3}$, and $c_{4}$ and therefore also $c_{5}$ can only be determined up to factor. Keeping $c_{2}$ fixed, we can determine the other coefficients and the exponent by fitting to existing transport data, for TC flow to the torque measurements of [14-18], and for pipe flow to the friction factors as function of $R e$ documented in [19]. Once the coefficients are given, $N u$ is prescribed and eqns $(9,10)$ are solved numerically for the Reynolds numbers $R e$ of the base flow and $R e_{w}$ of the wind.

As Fig. 2 shows, the parameters $c_{1}=0.435, c_{2}=29.3, c_{3}=0.220, c_{4}=0.0147, c_{5}=$ $1.99 \cdot 10^{-6}$ and $\gamma=1.23$ describe the overall behaviour of the friction factor $f=N u / R e$ vs. $R e$ for TC quite well. However, as in the case of RB flows, much more stringent information is contained in plots of the relative error (lower left frame of Fig. 2). The relative error remains within a few percent. For comparison we also show in both diagrams the results for a fit suggested by the Prandtl-von Karman formula, $1 / \sqrt{f}=A \ln (\operatorname{Re} \sqrt{f})+B$ with adjustable parameters $A$ and $B$. The differences between the functions show up in the reduced plot: while the error for the logarithmic profile seems to be bend systematically, the new fit is constant within error bars. 
The behaviour for pipe data is similar. A fit to the data of Smits and Zagarola [19] gives the parameters $c_{1}=0.000927, c_{2}=0.003, c_{3}=0.888, c_{4}=0.0104, c_{5}=0.00124$, and $\gamma=1.131$. Fig. 2 shows the fit to the friction factor $f=(R \Delta p / \ell) /\left(U^{2}\right)$ (top right) and the relative error (lower right). The deviations between the current model, the Prandtl-von-Karman model, and the actual data are of about the same magnitude. Hence, within the uncertainty of the data, both models give a consistent representation of the data. A more detailed discussion of these data will be presented in forthcoming publications [20,21].

Conclusions: The analysis shows how the thermal system and the shear flows can be discussed on the same footing once the relevant flux and the dissipation rate of the transporting 'wind' that contributes to the transverse flux are identified. Copying the boundary type and bulk/fluctuation models for the scaling behavior from RB flow, the corresponding relations for the fluxes and the energy dissipation rates can be obtained. An analysis of existing data suggests that the transition between the different regions occur at different Reynolds numbers for the flux and the energy dissipation rate, giving rise to at least four different regimes in flow behavior which contribute with different weights. Therefore, also in TC and in pipe flow no pure power law behavior for the fluxes or dissipation rates can be expected.

Financial support by DFG and FOM is gratefully acknowledged.

\section{REFERENCES}

[1] S. Grossmann and D. Lohse, J. Fluid Mech. 407, 27-56 (2000); Phys. Rev. Lett. 86, 3316-3319 (2001); Phys. Rev. E 66, 016305/1-6 (2002); Phys. of Fluids 16, 4462-4472 (2004).

[2] G. Ahlers, Phys. Rev. E 63, 015303(R) (2000).

[3] S. Chaumat, B. Castaing, and F. Chilla, In Advances in Turbulence IX (ed. I. P. Castro \& P. E. Hancock), Barcelona: International Center for Numerical Methods in Engineering, CIMNE (2002).

[4] R. Verzicco, Phys. of Fluids 16, 1965 (2004).

[5] E. Brown, D. Funfschilling, A. Nikolaenko, and G. Ahlers, Phys. Fluids 17, 075108 (2005).

[6] G. Ahlers, E. Brown, D. Funfschilling, S. Grossmann, and D. Lohse, Non-Oberbeck-Boussinesq effects in strongly turbulent Rayleigh-Bénard convection, J. Fluid Mech., submitted (2005).

[7] B. Eckhardt, S. Grossmann, and D. Lohse, Eur. Phys. J. 18, 541-545 (2000).

[8] B. Dubrulle and F. Hersant, Eur. Phys. J. B 26, 379-386 (2002).

[9] P. Bradshaw, J. Fluid Mech. 36, 177-191 (1969).

[10] B. Eckhardt, S. Grossmann, D. Lohse, Energy and Dissipation Balances in Rotating flows in Progress in Turbulence, Eds. J. Peinke, A. Kittel, S. Barth, M. Oberlack, Springer Proceedings in Physics 101, Springer, Berlin etc., 2005, 47-50.

[11] B. Dubrulle, O. Dauchot, F. Daviaud, P.-Y. Longaretti, D. Richard and J.-P. Zahn, Phys. Fluids 17095103 (2005).

[12] H. Faisst and B. Eckhardt, Phys. Rev. Lett. 91, 224502 (2003).

[13] B. Hof, C.W. van Doorne et al., Science 305, 1594 (2004).

[14] D. P. Lathrop, Ph. D. dissertation, The University of Texas at Austin, 1992.

[15] D. P. Lathrop, J. Fineberg, and H. L. Swinney, Phys. Rev. Lett. 68, 1515-1518 (1992).

[16] D. P. Lathrop, J. Fineberg, and H. L. Swinney, Phys. Rev. A 46, 6390-6405 (1992).

[17] G. S. Lewis and H. L. Swinney, Phys. Rev. E 59, 5457-5467 (1999).

[18] F. Wendt, Ingenieurs-Archiv 4, 577-595 (1933).

[19] M. V. Zagarola and A. J. Smits, Phys. Rev. Lett. 78, 239-242 (1997).

[20] B. Eckhardt, S. Grossmann, D. Lohse, Torque scaling in turbulent Taylor-Couette flow between independently rotating cylinders, submitted.

[21] B. Eckhardt, S. Grossmann, D. Lohse, Scaling of skin friction in turbulent pipe flow, in preparation. 